\title{
Consultations in Child Abuse Pediatrics
}

\author{
Author Names: \\ Ralph A. Hicks, MD; Antoinette L. Laskey, MD; Tara L. Harris, MD; Roberta A. \\ Hibbard, MD
}

\section{Author Affiliations:}

Department of Pediatrics, Indiana University School of Medicine, Riley Hospital for Children, Indianapolis, Indiana, USA. Dr. Laskey is now with the Department of Pediatrics, University of Utah School of Medicine, Salt Lake City, Utah, USA.

\section{Corresponding Author:}

Ralph A. Hicks, MD, Child Protection Programs, Department of Pediatrics, Indiana University School of Medicine, Riley Hospital for Children, 575 Riley Hospital Drive, Room XE 040, Indianapolis, Indiana USA 46202. Email raahicks@iu.edu. Telephone $317-274-2617$.

\section{Keywords:}

Child abuse, child abuse pediatrics, child maltreatment, consultation, pediatric subspecialty practice

This is the author's manuscript of the article published in final edited form as:

Hicks, R. A., Laskey, A. L., Harris, T. L., \& Hibbard, R. A. (2020). Consultations in Child Abuse Pediatrics. Clinical Pediatrics, 59(8), 809-815. https://doi.org/10.1177/0009922820920019 


\begin{abstract}
This study describes a hospital-based child abuse pediatrics (CAP) consultation service. Medical records for all CAP consultations during 2006-2009 were reviewed. Descriptive statistics were used for data analysis. Of 2495 consultations, 13 were excluded due to insufficient information, 1682 were examinations for suspected sexual abuse, and 800 were examinations for non-sexual abuse concerns. Among the latter group of 800 patients, the most common reasons for consultation were fracture $(33.5 \%)$, non-burn skin injury (16.8\%), burn (15.4\%), and intracranial injury (13.2\%). Median patient age was 11 months (range 3 days -16 years). Case fatality rate was 3.9\%. Final diagnosis was classified as definite/likely abuse in $40.0 \%$, questionable/unknown in $24.5 \%$, definite/likely accident in $23.6 \%$, no injury in $4.6 \%$, neglect in $4.0 \%$, and a medical condition in $3.2 \%$. Therefore, among consultations requested for suspected child maltreatment, a child abuse pediatrician concluded that abuse was definite or likely in less than half of patients.
\end{abstract}




\section{Introduction}

Child abuse and neglect is a common problem in the United States. During the 2017 federal fiscal year there were 4.1 million referrals made to child protective services (CPS) agencies, and an incidence for substantiated cases of 9.1 per 1,000 children. ${ }^{1}$ The physical and mental health costs of child abuse and neglect, both during childhood and later in life, have been well documented..$^{2-5}$ The financial costs of child maltreatment are staggering and have been documented..$^{6-10}$

In 2005 the American Board of Pediatrics approved a petition to begin a new subspecialty named child abuse pediatrics (CAP). ${ }^{11}$ The first subspecialty board examination in CAP was offered in 2009. This development has resulted in fellowship programs becoming standardized in terms of duration and basic requirements, as they now require accreditation by the Accreditation Council for Graduate Medical Education. The evolution of CAP as a subspecialty has paralleled reports documenting that practicing pediatricians feel undertrained and often uncomfortable diagnosing and managing child maltreatment. ${ }^{12}$ This is understandable given the recent rapid expansion in the published body of knowledge about this topic. ${ }^{13}$

The actual clinical practice of CAP has not been well described. Other pediatric subspecialties have attempted to define their clinical practice in order to plan curricula for undergraduate, graduate and continuing medical education programs. ${ }^{14}$ Such descriptions 
may also be useful for department chairs and hospital administrators who must plan for clinical service needs. In addition to providing direct patient care, child abuse pediatricians commonly participate in multidisciplinary team case reviews, provide formal and informal case reviews for investigators, provide telephone consultation for health care providers, are called upon for expert testimony in legal proceedings (civil and criminal), participate in community child abuse prevention and child safety initiatives, and provide education to professionals in a variety of disciplines. The purpose of this report is to describe the clinical practice of a hospital-based CAP consultation service.

\section{Methods}

The Institutional Review Board of Indiana University approved this study (Protocol \# 1011003548 | 0910-54) under an expedited category, with a waiver of informed consent.

Subjects for the study were patients for whom a formal CAP consultation was provided, including examination of the patient, during the period from January 1, 2006 through December 31, 2009 (inclusive). Patients for whom a child abuse pediatrician was called with questions and/or a record review was performed, but the patient was not examined, were not eligible for the study. Patients were identified from the CAP patient and billing logs for all consultations performed in the inpatient units, emergency department (ED), and outpatient clinics at a university and children's hospital-based child protection program. Most inpatient and ED consultations were performed at a tertiary care referral children's hospital and the only full service children's hospital in the state (population 
$6,483,802$ according to the 2010 census:

https://www.census.gov/content/dam/Census/library/publications/2011/dec/c2010br-

01.pdf). A smaller number of consultations was performed at 2 affiliated teaching

hospitals, 1 being the county hospital; both are Level I Trauma Centers and teaching

hospitals. Sexual abuse examinations were performed at an outpatient clinic or the ED of the children's hospital. During the study period, the total number of pediatric inpatient beds was approximately 490 . Total annual emergency department visits involving pediatric patients during the study period was approximately 52,775 , of which about half were seen at the children's hospital.

The hospitals' policies and procedures during the study period mandated that hospital social work be consulted in all cases of suspected child abuse or neglect. CAP consultation was not mandatory; however, CAP was often notified regarding patients about whom a report to CPS was made even if a consult was not formally requested. With respect to suspected child neglect, CAP was usually notified of such cases when a report was made to CPS, but not routinely asked to provide formal consultation, as was usually the case for other forms of child maltreatment. Any hospital staff, not just physicians, could consult CAP.

For each consultation, the following patient data was abstracted: demographic information, hospital and clinical unit where consultation was performed, reason for consultation, whether patient was new or known to the child protection team, whether a report was made to CPS, physical exam findings, other consultations, and final diagnosis. 
Specific laboratory tests and radiologic examinations noted in the consultation or recommended by the consulting physician were recorded, but not the results of individual tests.

For non-sexual abuse consultations, medical determinations of child abuse or neglect were based on all available medical evidence, and did not reflect a CPS or law enforcement disposition. A total of 11 diagnostic categories was utilized. The first seven diagnostic categories describe likelihood of physical abuse based on previously described criteria. ${ }^{15-17}$ The remaining four diagnostic categories were utilized for cases that did not apply to the above classification.

1. Definite abuse was diagnosed if any of the following were present: perpetrator confession, eyewitness, positive skeletal survey (unexplained occult fractures, fractures of various ages), other types of injury characteristic of abuse (e.g., patterned skin injuries, unexplained internal injuries), or a suspicious injury later followed by definite abuse.

2. Likely abuse was diagnosed if the patient's presenting injury was considered suspicious for abuse by the treating physicians and the history offered was inconsistent (i.e., implausible or no history, changing history, or delay in seeking care).

3. Questionable abuse was diagnosed when an injury was not considered suspicious or was of uncertain cause but the history offered was inconsistent, insufficient to explain the injury, or there was an inappropriate delay in seeking care. 
4. Unknown was assigned for cases with insufficient information in the medical record to allow for a determination.

5. Questionable unintentional injury was diagnosed for an isolated injury/incident plus either no history known, a history consistent with the type but not the extent of injury, or neglect involved.

6. Likely unintentional injury was diagnosed for an isolated injury with a consistent history, an injury consistent with the history offered but with neglect involved, an isolated injury with a minimal though consistent history, or a history consistent with the injury with aggressive or irresponsible behavior involved yet the injury was not directly inflicted.

7. Definite unintentional injury was diagnosed for motor vehicle crashes, pedestrian struck by an automobile, or multiple witnesses documented (e.g., police or emergency medical services at the scene).

8. Underlying medical conditions rather than injury.

9. Siblings of index patients examined to determine whether there was any injury or sign of neglect.

10. Suspected neglect.

11. Examination for suspected sexual abuse.

For this study, the diagnosis of physical abuse was made for patients who were classified in either the definite abuse or likely abuse categories ( 1 and 2 above). The diagnosis of unintentional injury was made for patients who were classified in either the definite unintentional or likely unintentional categories ( 6 and 7 above). The questionable abuse, 
unknown, and questionable unintentional categories (3-5 above) were diagnosed as indeterminate.

Examinations for suspected sexual abuse often have normal or nonspecific findings, in contrast to other types of maltreatment in which a diagnosis may be made based on the medical findings in conjunction with the history. ${ }^{18}$ Therefore, consultations for suspected child sexual abuse were analyzed separately.

The reason for consultation was easily identifiable so was limited to one (primary) reason. Subjects frequently had more than one final diagnosis and therefore all were listed.

Injuries to the head were classified as follows. Bruising to the head, without associated skull fracture or intracranial injury, was classified as bruising. Skull fractures, if an isolated finding (i.e., with no associated intracranial injury), were classified as a fracture. Intracranial hemorrhage without another underlying identified cause (e.g., coagulopathy, arteriovenous malformation) and hypoxic-ischemic injury were classified as intracranial injury. Intracranial hemorrhage, including subdural and subarachnoid, was not detailed further (e.g., by location or pattern) during the chart reviews.

Descriptive statistics were used for data analysis.

\section{Results}


For the 4-year study period, 2,495 consultations were identified. Thirteen cases were excluded because insufficient information was available to review. Consultations for suspected sexual abuse totaled 1,682. All other consultations totaled 800. During the study period, the program included 2-3 child abuse pediatricians (full-time equivalent total $1.25-2.25$ ). All of the child abuse pediatricians became board certified at the first offering of the subspecialty board examination in November 2009.

Of the 1,682 sexual abuse examinations, 1,062 (63.1\%) were scheduled examinations in the outpatient clinic and $620(36.9 \%)$ were acute examinations performed in either the ED or clinic, usually with forensic evidence collection. Data for outpatient clinic patients examined for suspected sexual abuse were not maintained in a way that allowed for analysis of demographic data and examination findings, and by the time of the study access to the medical records was not consistently available. Therefore, additional analysis of data for this group was not possible.

Of the 800 consultations for non-sexual abuse concerns, the median patient age at the time of examination was 11 months (range 3 days -16 years). The largest number of consultations was performed in the emergency department (46.2\%). Other locations where consultations were provided included the general and subspecialty inpatient units (27.9\%), pediatric intensive care unit (12.1\%), burn unit (11.0\%) and outpatient clinics $(2.8 \%)$. 
Reasons for requesting consultation are displayed in Table 1. The most common reasons were fracture (33.5\%), non-burn skin injury (16.8\%), burn (15.4\%), and intracranial injury (13.2\%). CPS was involved in $91.4 \%$ of cases.

Physical examinations were abnormal in $77.1 \%$ of patients. Of 571 patients $<2$ years of age, a skeletal survey was performed in $90.2 \%$ and head imaging in $81.4 \%$. At least 1 additional subspecialty consultant was involved in $75.4 \%$ of cases. The most common additional subspecialties involved were ophthalmology, neurosurgery, orthopedic surgery, general pediatric/trauma surgery and plastic surgery/burn service.

Many patients had more than one final diagnosis, reflecting multiple findings and/or injuries. Therefore, the percentage total of final diagnoses exceeded 100 (Table 2). The most common final diagnoses were fracture (36.8\%), bruise or other non-burn skin injury (27.5\%), intracranial injury (20.8\%), and burn (16.5\%).

Final diagnostic category was classified as definite or likely abuse in $40.0 \%$, questionable or unknown in $24.5 \%$, and definite or likely unintentional injury in $23.6 \%$ (Table 3 ). Medical conditions accounted for $3.2 \%$ and examples included apparent life-threatening event (now known as brief resolved unexplained event), sudden unexpected infant death, skeletal disorders (e.g., osteogenesis imperfecta, rickets), coagulopathies, infections, metabolic disorders, birth trauma, intracranial lesions (e.g., arteriovenous malformation), and ingestion/drug exposure. 
Among consultations for non-sexual abuse concerns, the case fatality rate was 3.9\% (32 of 800). Of the 32 fatalities, 18 were classified as due to abuse, 7 were classified as unintentional injury, 4 questionable/unknown, 2 due to underlying medical conditions, and 1 due to neglect. Of the fatalities classified as due to abuse, all were due to abusive head trauma. All 7 unintentional deaths involved asphyxial injury. The primary diagnoses associated with fatalities were intracranial injury (21), asphyxia (7), medical/surgical conditions (3), and burn due to a house fire (1). The 7 cases involving asphyxia included 5 in which unsafe sleep conditions were present by history, and one each due to choking and drowning. Of the 3 cases involving medical/surgical conditions, 2 were patients with multiple congenital anomalies, developmental delays and failure to thrive. The third patient, born with gastroschisis and fed via gastrostomy tube, was reportedly found unresponsive at home and presented with cerebral edema and intracranial hemorrhages. No information from the coroner, autopsy or investigators was available and the case was classified as unknown with respect to likelihood of abuse.

Diagnostic categories relating to likelihood of abuse by reason for consultation are depicted in Table 4. In contrast to the most common reasons for consultation, bruising and other non-burn skin injury was most likely to be classified as abusive (59.0\%) and least likely to be classified as unintentional injury (10.4\%). Burns were more likely to be classified unintentional injury than abusive (37.4\% vs. $26.8 \%$ of cases, respectively). Cases involving fractures and intracranial injuries had less extreme differences between the proportions judged abusive and unintentional injury. While $44.8 \%$ of intracranial injuries and $42.5 \%$ of fractures were classified as abusive, the remainder of cases in both 
groups were almost equally divided between unintentional injury and undetermined (i.e. questionable abuse, unknown, or questionable unintentional injury).

As is also illustrated in Table 4, when the reason for consultation was intracranial injury, only in a minority of cases (44.8\%) was abuse diagnosed. Therefore, among a selective patient population in whom there were already concerns for child maltreatment as evidenced by a request for a child abuse pediatrics consultation, the consultant diagnosed abuse in less than half of cases. The same was true for patients when the reason for consult was a bony fracture or burn.

\section{Discussion}

This study provides the first description of medical consultations by child abuse pediatricians in the hospital setting. Among consultations for non-sexual abuse concerns, the most common reasons for consultation were fractures, skin injury (primarily bruising and burns) and intracranial injury. The case fatality rate was 3.9\%, with nearly two-thirds of fatal cases due to intracranial injury. The likelihood of abuse varied by reason for consultation, with non-burn skin injury being most likely to be diagnosed as abusive (59.0\% abusive; $10.4 \%$ unintentional injury) and burns most likely to be diagnosed as unintentional injury (37.4\% unintentional injury; $26.8 \%$ abusive) The relatively high percentage of child abuse consultations for burns that resulted in a diagnosis of unintentional injury is notable; this may reflect discomfort on the part of primary 
providers in assessing the etiology of burns, or it may reflect practice variation specific to the burn unit at the study institution.

One may question our choice of seven diagnostic categories describing the likelihood of physical abuse. This was done because there is no gold standard for making the diagnosis, no one diagnostic schema has been validated, and the divergent opinions demonstrated when experienced physicians evaluate hypothetical cases. ${ }^{19,20}$ It is precisely for this reason that researchers who have studied classification schemes stress the importance of multidisciplinary or peer review assessment. ${ }^{19,20}$ Multidisciplinary assessment in cases of suspected child abuse/neglect has been shown to increase the accuracy of the assessment. ${ }^{21-24}$

Descriptive data such as is presented here can be useful for hospital administrators, pediatric department chairs, and other pediatric educators including pediatrics residency and CAP fellowship program directors. For example, consultation and patient volume data may help administrators when considering physician and other staffing needs for this subspecialty. This data may be used for comparison and in conjunction with other available general and subspecialty pediatrics workforce data. ${ }^{25}$ Such data can also be used to guide development and periodic reassessment of training curricula for pediatrics residency and CAP fellowship programs.

Perhaps the most important finding of our study is that, in this select population of pediatric patients in whom medical providers already suspected or had concerns for child 
maltreatment, CAP consultants made a diagnosis of definite or likely abuse in only $40.0 \%$ of cases. The fact that CPS was involved in such a high proportion of cases (91.4\%) reflects the fact that in many cases reports to CPS were made prior to arrival at our hospital or CAP consultation. The medical diagnosis of child abuse/neglect is not a process to be taken lightly, and this finding demonstrates one aspect of the value added by CAP consultation. Over diagnosis, i.e. diagnosis of child maltreatment when in fact the medical findings are due to an unintentional injury, underlying medical condition, or other cause, may result in unnecessary CPS and/or law enforcement investigations, civil and criminal proceedings, and even removal of children from their homes. ${ }^{26}$ Conversely, a missed diagnosis of child maltreatment leaves a child in an environment where $\mathrm{s} / \mathrm{he}$ is at continued risk for further injury or even death. ${ }^{27,28}$ Of additional note is that, when consulted by CPS, child abuse pediatricians frequently have a lower level of concern for abuse/neglect than the examining physicians and CPS case workers. ${ }^{29,30}$ Another study though showed that child abuse experts recommended a report to CPS more frequently compared to primary health care providers. ${ }^{31}$ Our findings and those from the aforementioned studies likely reflect the significant focus of CAP practice on mechanisms and patterns of injury, biomechanical principles, and the differential diagnosis of the various presenting clinical problems which prompt consultations. ${ }^{32}$

Previous studies have described consultations provided by child abuse pediatricians specifically for CPS agencies. ${ }^{29,30}$ These studies showed that when CPS asked that a child abuse pediatrician review a case to provide a second medical opinion, the child abuse pediatrician's opinion frequently differed from the original medical assessment. In 
addition, Anderst et al. ${ }^{29}$ observed that in many cases, non-child abuse pediatricians even after initiating a report to CPS or being asked by CPS for an opinion - did not provide CPS with a diagnosis. This obviously leaves a CPS caseworker in a most difficult situation with respect to being able to make an assessment about their case (i.e., whether or not to substantiate abuse or neglect). Both physicians and CPS caseworkers benefit from information that each are able to provide the other. Physicians often need additional history that may help, for example, to adequately explain the patient's medical findings or injuries. CPS case workers need to know, for example, whether a child's medical findings represent injuries and if they are adequately explained by the history offered.

While all state child abuse reporting laws in the U.S. include health care providers as mandatory reporters of suspected abuse/neglect, a CAP may help determine at what point there is enough suspicion to warrant a report to CPS, and when and what medical evaluation is indicated. Of note in this study is the increase in number of cases with fractures (268 to 294), bruise/skin or soft tissue trauma (134 to 220), burns (123 to 132), and intracranial injury (107 to 166; Tables 1 and 2, respectively) found once CAP was involved. Perhaps in some cases the additional findings were known at the time consultation was requested. This data demonstrates though the value of a CAP consultation and following published recommendations concerning the appropriate evaluation for suspected physical abuse in children in order to enhance detection of injuries. $^{33}$ 
The focus of this study concerned formal consultations in the hospital setting by a CAP. As noted in the Introduction, the work of child abuse pediatricians typically involves numerous other activities. For example, it is common for child abuse pediatricians to perform case reviews at the request of CPS and/or law enforcement. Such case reviews typically involve review of photographs, medical records and other information provided by investigators, and in some cases, a formal written report is requested. This work is clearly a service for agencies throughout one's community and state, but require time and effort on the part of the CAP that is not reimbursed as are patient care activities. Some programs provide this in a more formal way than others do. For example, since 2008 our program's parent institution has had a formal contract with the state CPS agency to provide medical consultation for CPS case managers that currently accounts for more than 5,000 case reviews each year.

The primary strengths of this study include the relatively large number of subjects and the fact that there were multiple faculty/staff physicians during the time studied.

There are limitations to this study. Being a retrospective chart review, it is subject to the limitations of missing charts, incomplete documentation, and difficulty interpreting entries. There was no follow-up information from CPS and law enforcement investigations, or from most medical providers, in the medical records. In order to minimize the possibilities of circular reasoning and subjectivity in the interpretation of the records, previously published and detailed classification systems to define the likelihood of abuse were utilized. Finally, this study presents data concerning direct 
clinical care from only one CAP program, and may not necessarily be generalizable to other regions, states, hospitals or practices.

\section{Conclusion}

In summary, this study provides data concerning the direct clinical work of child abuse pediatricians that can help guide (1) educators in curriculum planning for all levels of medical education, and (2) hospital and pediatric department administrators in planning for service needs in this subspecialty. Of importance is that in a population where consultation was requested because abuse was already suspected or considered, a child abuse pediatrician concluded that abuse was definite or likely in less than half of patients. 


\section{Acknowledgement:}

Richard L. Schreiner, MD provided comments on an earlier draft of the manuscript and suggestions for its improvement.

\section{Funding:}

This research received no specific grant from any funding agency in the public, commercial, or not-for-profit sectors.

\section{Declaration of Conflicting Interests:}

The authors' institution has received payment for their time for expert witness court testimony provided in cases involving suspected child abuse for which they were subpoenaed to testify.

\section{Previous Presentation:}

This study was presented in part at The Ray E. Helfer Society 2013 Annual Meeting; April 17, 2013; Sonoma, California. 


\section{References}

1. U.S. Department of Health and Human Services, Administration for Children and Families, Administration on Children, Youth and Families, Children's Bureau. Child Maltreatment 2017. 2019; https://www.acf.hhs.gov/cb/research-datatechnology/statistics-research/child-maltreatment. Accessed March 4, 2019.

2. Felitti VJ, Anda RF, Nordenberg D, Williamson DF, Spitz AM, Edwards V, Koss MP, Marks JS. Relationship of childhood abuse and household dysfunction to many of the leading causes of death in adults. The Adverse Childhood Experiences (ACE) Study. Am J Prev Med. 1998;14(4):245-258.

3. Felitti VJ. Adverse childhood experiences and adult health. Acad Pediatr. 2009;9(3):131132.

4. Jonson-Reid M, Kohl PL, Drake B. Child and adult outcomes of chronic child maltreatment. Pediatrics. 2012;129(5):839-845.

5. Sege RD, Amaya-Jackson L. Clinical Considerations Related to the Behavioral Manifestations of Child Maltreatment. Pediatrics. 2017;139(4).

6. Currie J, Spatz Widom C. Long-Term Consequences of Child Abuse and Neglect on Adult Economic Well-Being. Child Maltreatment. 2010;15(2):111-120.

7. Fang X, Brown DS, Florence CS, Mercy JA. The economic burden of child maltreatment in the United States and implications for prevention. Child Abuse Negl. 2012;36(2):156165.

8. Gelles RJ, Perlman S. Estimated annual cost of child abuse and neglect. 2012. http://preventchildabuse.org/wp-content/uploads/2016/02/PCA_COM2012-1.pdf. Accessed January 19, 2018.

9. Irazuzta JE, McJunkin JE, Danadian K, Arnold F, Zhang J. Outcome and cost of child abuse. Child Abuse Negl. 1997;21(8):751-757. 
10. Rovi S, Chen PH, Johnson MS. The economic burden of hospitalizations associated with child abuse and neglect. Am J Public Health. 2004;94(4):586-590.

11. Block RW, Palusci VJ. Child abuse pediatrics: a new pediatric subspecialty. J Pediatr. 2006;148(6):711-712.

12. Lane WG, Dubowitz H. Primary care pediatricians' experience, comfort and competence in the evaluation and management of child maltreatment: do we need child abuse experts? Child Abuse Negl. 2009;33(2):76-83.

13. Schwartz KA, Preer G, McKeag H, Newton AW. Child maltreatment: a review of key literature in 2013. Curr Opin Pediatr. 2014;26(3):396-404.

14. Geggel RL. Conditions leading to pediatric cardiology consultation in a tertiary academic hospital. Pediatrics. 2004;114(4):e409-417.

15. Leventhal JM, Thomas SA, Rosenfield NS, Markowitz RI. Fractures in young children. Distinguishing child abuse from unintentional injuries. Am J Dis Child. 1993;147(1):8792.

16. Thomas SA, Rosenfield NS, Leventhal JM, Markowitz RI. Long-bone fractures in young children: distinguishing accidental injuries from child abuse. Pediatrics. 1991;88(3):471476.

17. Feldman KW, Bethel R, Shugerman RP, Grossman DC, Grady MS, Ellenbogen RG. The cause of infant and toddler subdural hemorrhage: a prospective study. Pediatrics. 2001;108(3):636-646.

18. Jenny C, Crawford-Jakubiak JE, Committee on Child Abuse and Neglect. The evaluation of children in the primary care setting when sexual abuse is suspected. Pediatrics. 2013;132(2):e558-567.

19. Laskey AL, Sheridan MJ, Hymel KP. Physicians' initial forensic impressions of hypothetical cases of pediatric traumatic brain injury. Child Abuse Negl. 2007;31(4):329342. 
20. Lindberg DM, Lindsell CJ, Shapiro RA. Variability in expert assessments of child physical abuse likelihood. Pediatrics. 2008;121(4):e945-953.

21. Bross DC, Ballo N, Korfmacher J. Client evaluation of a consultation team on crimes against children. Child Abuse Negl. 2000;24(1):71-84.

22. Hochstadt NJ, Harwicke NJ. How effective is the multidisciplinary approach? A followup study. Child Abuse Negl. 1985;9(3):365-372.

23. Jaudes PK, Martone M. Interdisciplinary evaluations of alleged sexual abuse cases. Pediatrics. 1992;89(6 Pt 2):1164-1168.

24. Wolfteich P, Loggins B. Evaluation of the children's advocacy center model: efficiency, legal and revictimization outcomes. Child and Adolescent Social Work Journal. 2007;24(4):333-352.

25. American Board of Pediatrics. Pediatric Physicians Workforce Data Book. 2018; https://www.abp.org/sites/abp/files/pdf/pediatricphysiciansworkforcedatabook20172018.pdf. Accessed September 7, 2018.

26. Kirschner RH, Stein RJ. The mistaken diagnosis of child abuse. A form of medical abuse? Am J Dis Child. 1985;139(9):873-875.

27. Jenny C, Hymel KP, Ritzen A, Reinert SE, Hay TC. Analysis of missed cases of abusive head trauma. JAMA. 1999;281(7):621-626.

28. Sheets LK, Leach ME, Koszewski IJ, Lessmeier AM, Nugent M, Simpson P. Sentinel injuries in infants evaluated for child physical abuse. Pediatrics. 2013;131(4):701-707.

29. Anderst J, Kellogg N, Jung I. Is the diagnosis of physical abuse changed when Child Protective Services consults a Child Abuse Pediatrics subspecialty group as a second opinion? Child Abuse Negl. 2009;33(8):481-489.

30. McGuire L, Martin KD, Leventhal JM. Child abuse consultations initiated by child protective services: the role of expert opinions. Acad Pediatr. 2011;11(6):467-473. 
31. Sege RD, Flaherty E, Jones R, Price LL, Harris D, Slora E, Abney D, Wasserman R, Child Abuse R, Experience Study Study T. To report or not to report: examination of the initial primary care management of suspicious childhood injuries. Acad Pediatr. 2011;11(6):460-466.

32. American Board of Pediatrics. Content Outline: Child Abuse Pediatrics. 2017; https://www.abp.org/sites/abp/files/pdf/chab.pdf Accessed September 7, 2018.

33. Christian CW, Committee on Child Abuse and Neglect. The evaluation of suspected child physical abuse. Pediatrics. 2015;135(5):e1337-1354. 
TABLE 1. Reasons for Requesting Child Abuse Pediatrics Consultations ( $\mathrm{N}=800$ )

Reason For Consult

Fracture

Bruise/skin or soft tissue trauma

Burn

Intracranial injury

Apparent life-threatening event

Other injury

Sibling injured

Fall

Failure to thrive

Ingestion

Respiratory distress

Neglect

Eye injury

Seizure

Vomiting

Total
Patients (\%)

$268(33.5)$

$134(16.8)$

$123(15.4)$

$107(13.4)$

$39(4.9)$

$33(4.1)$

$33(4.1)$

$18(2.2)$

$11(1.4)$

$8(1.0)$

$8(1.0)$

$6(0.8)$

$5(0.6)$

$5(0.6)$

$2(0.2)$

800 (100) 
TABLE 2. Final Diagnoses for Child Abuse Pediatrics Consultations

\section{Final Diagnosis}

Fracture

Bruise/skin or soft tissue trauma

Intracranial injury

Burn

Retinal hemorrhages

Normal examination

Failure to thrive

Other $^{b}$

Developmental delay

Hypoxic-ischemic encephalopathy

Infection

Metabolic disorder

Apparent life-threatening event

Epilepsy

\section{Patients (\%)}

$294(36.8)$

$220(27.5)$

$166(20.8)$

$132(16.5)$

$41(5.1)$

$38(4.8)$

$26(3.2)$

$19(2.4)$

$17(2.1)$

$17(2.1)$

$10(1.2)$

$10(1.2)$

$7(0.9)$

$6(0.8)$

${ }^{a}$ Because patients could have more than one final diagnosis, the percentage total exceeds 100.

b “Other" includes a variety of specific medical, surgical and mental health conditions not listed elsewhere in this Table. 
TABLE 3. Diagnostic Category among Patients Evaluated by Child Abuse Pediatrics Consultant

\section{Diagnostic Category}

Definite or likely abuse

Questionable or unknown

Definite or likely unintentional injury

No injury/sibling exam (normal)

Neglect

Medical conditions

\section{Patients (\%)}

$320(40.0)$

$196(24.5)$

$189(23.6)$

37 (4.6)

$32(4.0)$

$26(3.2)$

Total

800 (100) 
TABLE 4. Diagnostic Category by Reason for Consultation

\begin{tabular}{|c|c|c|c|}
\hline \multirow{2}{*}{ Reason for Consult } & Unintentional & Questionable or & Abuse \\
& Injury & Unknown & (Definite/Likely) \\
\hline Burn & $37.4 \%$ & $30.9 \%$ & $26.8 \%$ \\
\hline Fracture & $28.7 \%$ & & $42.5 \%$ \\
\hline Intracranial Injury & $21.5 \%$ & $25.4 \%$ & $44.8 \%$ \\
\hline Non-Burn Skin & $10.4 \%$ & & $59.0 \%$ \\
\hline Injury & & & \\
\hline
\end{tabular}

${ }^{a}$ Row totals do not equal $100 \%$ because the following diagnostic categories are not included: cases involving primarily neglect, those with underlying medical conditions rather than injury, and siblings of index patients examined primarily for a wellness check. 